\title{
Fundamental Research in Cosmoparticle Physics and the Visualisation: Symbiosis of graphics and conceptualisation
}

\author{
Kristina Mettus \\ Krylo Bobra Media, \\ 62 Jukova str, apt 43, Moscow 123103, Russia \\ kmettus@gmail.com
}

\author{
Anna Nazarova \\ 17 De Vere Mews, \\ London W8 5AL, United Kingdom \\ annanazo@gmail.com
}

\section{INTRODUCTION}

Cosmoparticle physics is a science, which is varied and multifaceted in ideas and figures. According to the definition of cosmoparticle physics by Maxim Kholpov, Director of Virtual Institute of Astroparticle physics in Paris, "in the proper combination of the indirect physical, astrophysical and cosmological signatures", cosmoparticle physics allows the study of the theory of everything and the true history of the universe. This science requires accurate calculations, data collection, and its succinct and visual interpretation. Cosmoparticle science brings together macro-scale objects and particle-scale objects, which are not within the physical capability of the eye. There has always been a need to allow intangible and unencompassable objects to be visualised. This tendency has been defined by a human's capacity of thinking abstractedly, which allows visualising the ideas, non-figurative notions or notions that could be described through theoretical means. Communication design serves humanity's needs for cosmoparticle identification.

\section{SCIENTIFIC DATA VISUALISATION}

Many attempts have been undertaken to bring "Big Science" to a wider audience. In educational documentaries, through application of graphic design to science, it is possible to deliver scientific data to a larger audience.

Most inspirational examples can be found in the educational cinema of the late Soviet era (Thaw era) which turned to the achievements of the feature film-making of the 1920s and developed a language of signs based on automatic significations (montage, slow-motion and fast-motion effects, extreme low- and high-angle framing, reverse motion, etc.).
The distinguishing feature of this cinema style is that despite being completely technical and having at its disposal a system of lenses and other gadgets, this cinema creates a realm of possible worlds and not only reflects reality but also builds its own visual entity, deriving its power over viewers from their previous cinematic or visual life experiences.

Soviet documentaries attempted to visualise invisible processes in the natural world, which required using a cinematic language that is quite different from graphic motion simulations of scientific events achievable today. Even though no photographs of particle events or star clusters or dust lanes in elliptical galaxies were possible, human creativity in visualisation was abundant. Experimentation with cinematography, stop-motion animation, or still graphics, was expertly used by the Soviet cinematographer Vladimir Kobrin, who having been inspired by Dali, pop art, and kinetics, tried to use experimental methods in cinematography and graphics to explain radioactivity, nuclear energy, the physical basics of quantum theory, and many other fundamental physics-related issues. Experiments of Kobrin implied using an especially intricate means of expression to intensify insights into the world.

Nowadays, because of the strong technological advancement of humankind, there are many ways of visualising cosmoparticle physics, like using telescopes to take pictures of galaxies and using computers to detect particle collisions and measure their actions. One of the most significant discoveries in the field of particle physics which is related to cosmoparticle physics is the Higgs boson. A group of documentarians, headed by theoretical physicist David Kaplan, grasped the urge for explaining the phenomenon of the the Higgs boson and rediscovering the collective 
international scientific effort to detect it, made a documentary entitled "Particle Fever". To tackle the challenge of visualising the groundbreaking scientific discovery made in 2012, David Kaplan collaborated with the MK12 design bureau to make the opening credits, which used motion design based on the original photography of particle physics events to introduce the viewer to the subject of particle physics.
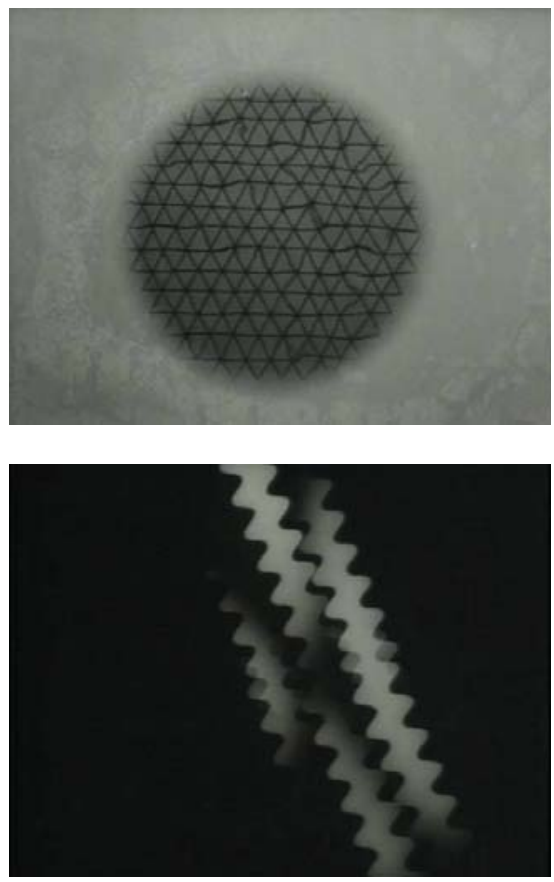

Figure 1: Vladimir Kobrin, "The Phenomenon of Radioactivity”, 1977

MK12 found inspiration in the actual dataset visuals that were coming out of the Large Hadron Collider. Those images were the springboard into the kinetic space in which MK12 were experimenting. At the same time, MK12 admitted that in making motion patterns of the Higgs boson's movement, they were closer to the theoretical viewpoint, in creating visual metaphors for what they call "very heady giant ideas".

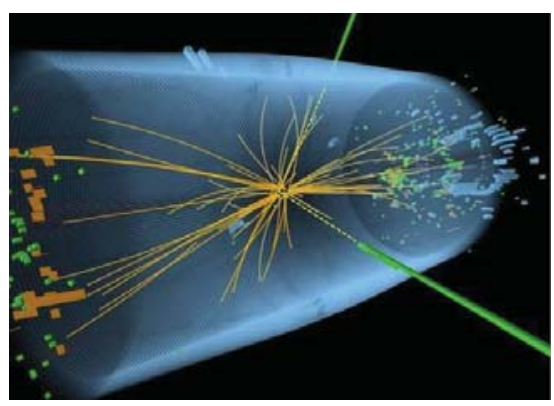

Figure 2: Traces from a collision in the Compact Muon Solenoid detector of the Large Hadron Collider

\section{CONCLUSION}

Graphic design is skillfully used in representing scientific data, which only reasserts its genuine function to summarise diverse data as symbolic images and elaborate forms and their proportions into plots and narrative structures suitable for aesthetic representation. Through graphic visualisation, data may be powerfully enhanced to convert it into accurate representations and abstract ideas. Cosmoparticle physics has been one of the fields of fundamental science that is based on processes inaccessible to a human eyesight and through popular lense systems. It has been one of the last to be visualised through unique technical equipment and scientific projects like the Hubble telescope and the Compact Muon Solenoid detector.

Nevertheless, astroparticle physics has been subject to reinterpreting and enabling visual comprehensibility to people well before the technical advancement of high-tech photographing and imaging, which required imagination and usage of aesthetical as well as semiotic approaches. But even after recent technical advances, there are phenomena like dark matter that are still inaccessible to photography, as well as lying beyond complete human understanding. Urged to represent accurate data, graphic design does not forget about augmentation of pure fundamental physics through interpretation when the latter finds itself contextualised. Finally, at the moment when physics expands into the sphere of society, it is bringing a new range of socio-economical issues to the surface, where graphic design, communicative design, and lettering, become extremely relevant.

\section{REFERENCES}

Massey, R.J., Christensen, L.L., and Frankel, F. (2007) Dark matter comes to light, American Scientist, Sigma Xi, The Scientific Research Society.

http://www.americanscientist.org/issues/pub/2007/ 5/dark-matter-comes-to-light (retrived 26 May 2014).

Maxim Yu. Kholopov, M.V. (2004) Cosmoparticle physics - the challenge for the millenium, In H.V. Klapdor-Kleingrothaus (ed.), Beyond the Desert 2003, Springer, Proceedings in Physics, Vol. 92, pp 239-248.

Zlydneva, N. (2008) The trace of the avant-guarde in Soviet educational cinema. Studies in Environmental Aesthetics and Semiotics VII, pp. 75-83.

http://www.eki.ee/km/place/pdf/kp7 05 zlydneva.p df (retrived 26 May 2014). 